Géopolitique de la connaissance et transferts culturels

\title{
Géopolitique de la connaissance et transferts culturels
}

Florent VILLARD

\section{CpenEdition}

Journals

Édition électronique

URL : http://journals.openedition.org/transtexts/506

DOI : 10.4000/transtexts.506

ISSN : 2105-2549

Éditeur

Gregory B. Lee

Référence électronique

Florent VILLARD, «Géopolitique de la connaissance et transferts culturels », Transtext(e)s

Transcultures 跨文本跨文化 [En ligne], 9 | 2014, mis en ligne le 16 octobre 2015, consulté le 19 avril

2019. URL : http://journals.openedition.org/transtexts/506 ; DOI : 10.4000/transtexts.506

Ce document a été généré automatiquement le 19 avril 2019

(c) Tous droits réservés 


\section{Géopolitique de la connaissance et transferts culturels}

Florent VILLARD

\section{Actualité mémorielle et politique de l'Institut franco- chinois de Lyon (1921-1946)}

1 L'écriture de l'histoire est toujours une intervention dans le présent. Nous publions ce volume thématique dans le contexte d'une irruption inédite, et spectaculaire, de l'Institut franco-chinois de Lyon (zhongfa daxue 中法大学) dans l'espace publique.

2 Négligé pendant plusieurs décennies après sa fermeture officielle en 1946, la riche et édifiante histoire de l'Institut avait refait surface, il y a une dizaine d'années, lorsque la ville de Lyon fit le choix de valoriser ce capital culturel en déshérence. Un lieu de mémoire fut crée dans les anciens locaux de l'Institut et une exposition dédiée à l'IFCL s'était tenue dans le pavillon Rhône-Alpes lors de l'Exposition universelle de Shanghai. Jusqu'en mars 2014, cet héritage franco-chinois jouait le rôle de produit d'appel pour attirer les touristes et les investisseurs chinois à Lyon. Dans le contexte de la redéfinition de l'identité de la ville envisagée désormais comme une marque dont il s'agit de vendre l'image, l'IFCL pouvait servir, à raison, de caution historique à un nouveau roman métropolitain visant à ancrer Lyon dans la mondialisation.

Mais les usages contemporains de l'IFCL ont subitement changé d'échelle lors de la visite d'Etat en France du Président Xi Jinping 习近平 en mars 2014. Dès lors, les aspects patrimoniaux et marchands circonscrits au cadre provincial de la capitale des Gaules furent supplantés par des enjeux de politique intérieure chinoise. La visite présidentielle à Lyon augurait d'un déplacement de perspective et d'une récupération de la mémoire du lieu. Les éléments de langage des médias chinois suggéraient une visite en forme de retour aux sources du Parti communiste en évoquant le passage à Lyon des dirigeants légendaires que sont Zhou Enlai 周恩来 et Deng Xiaoping 邓小平, au mépris de la réalité historique. Dans le même temps, la présence à Lyon de l'ancien ministre de la RPC Li Lisan 
李立三 en 1921 pour exiger un fonctionnement plus démocratique de l'Institut fut occultée par la presse chinoise.

4 On assista à une actualisation mémorielle spectaculaire qui visait à inscrire l'expérience de l'IFCL dans une histoire officielle du régime de Pékin et de son Parti unique. A l'époque, nous avions alerté les acteurs lyonnais sur les enjeux politiques et symboliques d'une telle récupération, inquiets d'une mémoire mise en spectacle par un Etat déterminé à contrôler chez lui, et maintenant chez les autres, les discours publics et les récits historiographiques le concernant. ${ }^{1}$

5 Au-delà de ses aspects symboliques, ces usages politiques de la mémoire appellent à conserver une vigilance absolue concernant la préservation des traces matérielles de cette histoire. Déposées à la Bibliothèque municipale de Lyon, les archives de l'ancienne Association franco-chinoise de Lyon appartiennent à L'Université Jean Moulin-Lyon 3. Elles se composent des archives administratives de l'Institut franco-chinois - témoignage de la vie sociale, culturelle et universitaire de ses 473 étudiants - et des 25000 ouvrages et près de 500 périodiques de sa bibliothèque. Cette dernière, constituée par et pour des étudiants chinois entre 1921 et 1946, représente une ressource documentaire unique pour les spécialistes de l'histoire culturelle de la Chine moderne.

Si les contributions de ce volume interviennent au sein d'une actualité mémorielle dense et confuse, les réflexions qui animent ces textes se situent à un autre niveau et dans une autre temporalité.

7 En 2015, la Chine est sur le point de devenir la première puissance mondiale. Elle prend la main. Mais c'est pour tourner la manivelle d'une boite à musique composée il y a plus de deux siècles en Europe. ${ }^{2}$ La Chine s'est appropriée progressivement, et dans la douleur, le langage et les pratiques d'une modernité eurocentrée: la techno-science, la maitrise prétendument « rationnelle» de la nature et des activités humaines, le capitalisme, les institutions de l'Etat-nation.

Ecole de la modernité, l'IFCL fut un laboratoire exemplaire de ce processus d'apprentissage, un lieu d'auto-colonisation cognitive et culturelle à l'usage des élites chinoises. Contre l'historicisme des acteurs de l'époque, il faut évidemment rappeler que cette expérience ne fut aucunement le résultat d'un stade historique nécessaire et inévitable du développement de la Chine. Elle s'inscrivait dans le cadre d'un projet politique et idéologique. La France souhaitait répandre sa science, sa culture et ses valeurs dans un pays déjà envisagé à l'époque comme un formidable marché économique potentiel.

Que les intellectuels chinois de la période du 4 mai, conquis par la science et le nationalisme, aient œuvré activement à ce projet de «modernisation» de la société chinoise ne fait cependant aucun doute. ${ }^{3}$ Anciens anarchistes et fervents républicains, les principaux acteurs chinois de l'IFCL, de Cai Yuanpei 蔡元培 à Li Shizeng 李石曾, avaient aussi adhéré à la dimension politique de la modernité lors de leurs séjours prolongés en Europe. Ils croyaient au projet d'autonomie politique qui s'était manifesté au moment de la Révolution française. Ils soutenaient la nécessité d'une auto-institution des sociétés modernes. Au côté de Mr Science (Sai xiansheng 赛先生), ils défendaient Mr Démocratie (De xiansheng 德先生). Cet héritage progressiste de la modernité se trouve aujourd'hui occulté par ceux qui, en Chine, réécrivent l'histoire du point de vue des vainqueurs, en l'occurrence de l'oligarchie bureaucratique au pouvoir à Pékin. 


\section{Une école de la modernité : Arts, littérature et science}

10 A la suite d'un premier atelier qui s'est tenu à Lyon en janvier 2012 sur le thème « Migrations des savoirs, le cas de l'IFCL », nous avons lancé, en 2014 (puis en 2015), un projet recherche collaboratif intitulé «Lyon Sino-French Institute Archives: A Cultural and Academic Heritage of Global Modernity in the Age of Digital Humanities ». Dans le cadre de ce projet qui vise à interroger la vie sociale et culturelle des étudiants, leur imaginaire intellectuel et leurs catégories épistémologiques, nous souhaitons organiser, cataloguer et numériser les archives de cet Institut. En rendant accessible ces archives numériques, nous faciliterons la réalisation d'autres travaux académiques sur le sujet et nous permettrons une meilleure diffusion de ces documents historiques auprès d'un public plus vaste. Une histoire sociale et culturelle de l'IFCL et de ses ramifications en Chine sera réalisée afin d'évaluer l'impact de cette expérience technologique, scientifique et culturelle. ${ }^{4}$ Le présent volume constitue une étape préliminaire dans cet effort collectif pour traiter scientifiquement ces archives et l'histoire dont elles témoignent.

Dans la contribution initiale de ce volume, Valentina De Monte, ancienne conservatrice du Fonds chinois de la Bibliothèque municipale de Lyon, offre un état des lieux de première main sur les collections de l'Institut. Soulignant la valeur unique de ces documents, elle rappelle l'histoire du Fonds et s'interroge sur la diversité de ses usages en relation avec la nature particulière de ces documents. A première vue, leur dimension très spécifique les destine à un public de spécialistes et de chercheurs. Mais la mission de service public d'une bibliothèque nationale implique aussi une nécessaire ouverture vers une audience plus large. Valentina De Monte précise que cette articulation entre les enjeux de la recherche et ceux de la diffusion culturelle devrait être prise en compte dans l'optique d'un projet de numérisation des archives et des imprimés.

12 En traitant l'épisode de "La Marche sur Lyon », Gregory Lee nous rappelle le contexte houleux qui présida à l'ouverture de l'Institut à l'automne 1921. Les intellectuels chinois imaginèrent l'IFCL comme une alternative à la formule "travail-études » qui avait été initiée dès 1909 par Li Shizeng. Ce mouvement dont l'objectif était de permettre à des étudiants chinois de financer leurs études en travaillant dans les usines françaises s'était considérablement développé à la fin de la première guerre mondiale avant d'être victime de la crise économique de 1921. Abandonnés à leur sort, les étudiants chinois en France vont faire face au chômage et au manque d'argent. L'établissement de l'IFCL en septembre 1921 leur offrait l'espoir de pouvoir rester en France et de réaliser des études dans des conditions plus dignes. Mais l'IFCL n'avait pas été conçu pour eux. Il devait accueillir des étudiants solvables, préalablement sélectionnés en Chine et indifférents au militantisme politique, à la différence de leurs camarades du Mouvement travail-études. En s'appuyant sur des sources de première main, Gregory Lee relate les incidents connus sous le nom de la « Marche sur Lyon » (Lida yundong 里大运动) du point de vue de l'appareil administratif français. Il narre avec une précision chirurgicale l'enchainement des évènements qui ont provoqué la détention et l'expulsion hors de France d'une centaine d'étudiants chinois.

Les contributions de Wang Yiyan et Jacqueline Estran nous confirment que l'expérience de l'IFCL s'envisage aussi du point de vue de l'histoire artistique et littéraire. Certains étudiants de l'Institut sont en effet devenus des écrivains et des artistes de renom. Wang Yiyan propose une contribution inédite sur le rôle de Lyon dans la constitution d'un art moderne chinois au XXe siècle. Elle démontre l'influence des écoles d'art françaises, et 
notamment l'Ecole Nationale des Beaux-Arts de Lyon (ENBAL), dans la formation des artistes chinois et la création d'un canon national moderne pour la Chine. Grâce à un travail méticuleux dans les archives de l'IFCL et de l'ENBAL, Wang Yiyan restitue le parcours et les hauts faits des plus talentueux de ces étudiants en art tels que Wang Jingyuan 王靜遠, Pan Yuliang 潘玉良, Chen Zhixiu 陳芝秀, Fang Yun 方蘊, Chang Shuhong 常書鴻, Lü Sibai 吕斯百, Wang Linyi 王臨Z, etc. Autant d'artistes qui joueront un rôle de premier plan dans le domaine de l'éducation artistique et esthétique en Chine. Wang Yiyan nous rappelle ainsi que le projet de modernisation des intellectuels chinois de la période du 4 mai 1919, et notamment du co-fondateur de l'IFCL Cai Yuanpei, ne se limitait pas aux dimensions technoscientifiques et politiques mais qu'il visait aussi le champ de l'esthétique.

Jacqueline Estran aborde l'histoire culturelle de l'IFCL à partir de la question du genre, en soulignant un aspect remarquable de cette institution, signature de sa modernité : la pr ésence de plusieurs dizaines d'étudiantes et, parmi celles-ci, un nombre non négligeable d'entre elles ayant réalisé un doctorat. L'article nous éclaire sur les origines géographiques et la sociologie de ces étudiantes. Il nous renseigne aussi, en détails, sur les réalisations scientifiques de Luo Zhenying 羅振英, Lin Baoquan 林賔權, Wu Xuxin 吳續新 et Liang Daozhen 梁導貞, toutes doctorantes de l'IFCL. Dans la seconde partie de cette étude, l'auteur propose une analyse biographique inédite de Su Xuelin 蘇雪林, étudiante de l'Institut qui deviendra par la suite une figure majeure de l'histoire de la littérature chinoise moderne. A travers les textes de Su Xuelin, et notamment son autobiographie, Jacqueline Estran nous révèle la complexité du parcours intellectuel de l'écrivaine et le rôle crucial joué par son séjour à Lyon dans ses choix de vie.

Les interventions de Marie-Julie Maitre et Liang Hongling abordent l'IFCL dans le temps long de la modernité autour de la géopolitique des savoirs, de la migration des idées et des questionnements historiographiques liés à l'eurocentrisme. Dans "Géopolitique du savoir et philosophie dans le contexte de l'Institut Franco-chinois de Lyon », Marie-Julie Maitre s'appuie sur les travaux d'Edward Said et de Walter Mignolo pour proposer une analyse audacieuse des thèses de philosophies des étudiants de l'IFCL dans le cadre d'une géopolitique coloniale de la connaissance. Elle interroge le statut de la philosophie chinoise dans le contexte d'une domination épistémologique de l' "Occident», en prenant soin de ne pas réduire cette catégorie à sa définition culturelle. Liang Hongling prolonge cette réflexion en soulignant l'occultation du contexte colonial dans lequel s'instituent les politiques de « coopération » et « d'entraide » entre la Chine et l'Occident dans le domaine éducatif à la fin du XIX siècle. A l'appui de sa démonstration, l'auteur s'intéresse aux usages politico-culturels de l'indemnité des boxers, et propose une étude comparée des programmes éducatifs américains et français à l'attention des étudiants chinois. L'essai offre une mise en perspective internationale salutaire de l'expérience de l'IFCL.

\section{Varia : François Jullien, la critique transculturelle et les enjeux transnationaux de la thèse du " dépassement de la modernité »}

De par l'ampleur de son œuvre, la singularité de son approche philosophique et ses talents de pédagogue, François Jullien est devenu incontournable dès lors que se trouve 
en jeu la question de l'histoire intellectuelle chinoise en rapport avec celle de l'Europe. Dans les sciences humaines, en France et au-delà, ses laudateurs se bousculent pour apprécier cette entreprise de relecture de la tradition philosophique occidentale à l'aune de la « pensée chinoise ». En réplique à l'opus sévère de Jean François Billeter (Contre François Jullien, Allia, 2006), l'auteur de Procès et création s'était défendu vigoureusement d'une lecture essentialiste de la Chine et des relations interculturelles, revendiquant une approche constructiviste de la différence qui serait étrangère à la vulgate culturaliste.

Dans un texte inédit en français, Fabian Heubel creuse la question de la différence culturelle et du comparatisme chez Jullien. ${ }^{5}$ Il propose une critique transculturelle efficace de l'approche jullieniste à partir d'un autre a priori historique, celui de la modernisation comme " problème partagé et commun ». Fabian Heubel nous rappelle que le choix méthodologique de Jullien n'est rendu possible qu'en faisant abstraction des réalités sociales et historiques modernes. En considérant une relative homogénéité de la haute culture lettrée dans la Chine impériale et en postulant une continuité philosophique et culturelle intrinsèque entre la Grèce ancienne et l'Europe des lumières deux a priori historiques déjà contestables - l'hypothèse méthodologique de l'extériorité $\mathrm{du}$ " texte chinois » apparait effectivement crédible. Mais il faudrait alors renoncer à intégrer l'expérience moderne de la Chine dans notre lecture du monde présent, au mépris des phénomènes de circulation et d'hybridation propre à la condition postcoloniale de l'histoire moderne. Fabian Heubel conteste la pensée de «l'écart» proposée par Jullien. Prenant au sérieux la " philosophie sinogrammatique moderne et contemporaine » dans sa dimension hybride et transculturelle, il anticipe, et appelle de ses vœux, une sinisation de la philosophie européenne.

Dans «The syndrom of overcoming modernity: learning from Japan about ultranationalism ", Alain-Marc Rieu propose une réflexion comparative stimulante sur les manifestations du nationalisme japonais au $\mathrm{XX}^{\mathrm{e}}$ siècle. Les tentatives intellectuelles de "dépassement de la modernité » réalisées dans le Japon en crise des années 1930s constituent le point de départ de l'essai d'Alain-Marc Rieu. Mais l'auteur ne s'en tient pas à une exégèse des actes de l' " infâme colloque » de Tokyo en juillet 1942, il interroge la radicalité de cette critique de la modernité en s'affranchissant du contexte spécifiquement japonais pour envisager cette «méta-idéologie » du « dépassement de la modernité » en tant que "syndrome d'un moment singulier et dangereux dans les modernisations des sociétés humaines ». Rieu nous rappelle que les manifestations de ce désenchantement des sociétés vis-à-vis de la modernité, qui engage en profondeur le rapport des contemporains à un passé mythique et proposent une vision a-historique des communautés nationales, fut aussi à l'œuvre en Allemagne et en France. Mais l'expérience japonaise du fascisme, ses ressorts idéologiques, sociaux et psychologiques, doit aussi nous éclairer sur des phénomènes discursifs et politiques à l'œuvre dans certaines sociétés contemporaines, notamment non-occidentales (Russie, Chine), qui envisage l'Europe en tant que "contre-modèle ». Ces tendances idéologiques actuelles suggèrent une modernité alternative ambigüe, et dangereuse, préservant une certaine transcendance et réinventant une continuité mythique avec une tradition et des racines pré-modernes. 


\section{NOTES}

1. Gregory Lee, Florent Villard, « A qui profite la visite du président chinois à Lyon », pages Idées, lemonde.fr, 25/03/2014. Page consultée le 26/03/2014, url : http:// www.lemonde.fr/idees/article/2014/03/25/dindon-laque-de-la-farce-lyon-accueille-lepresident-chinois-cui-bono_4389386_3232.html

2. Gregory Lee, « Le cadeau empoisonné de Versailles ou la Chine à la manivelle de l'orgue de barbarie ", Mouvements, 2012, 4, n72 (page consultée le 10/03/2014 url : http:// www.cairn.info/resume.php?ID_ARTICLE=MOUV_072_0079)

3. A la suite d'un Traité de Versailles infamant pour la Chine, les étudiants et les forces progressistes ont déclenché des manifestations de protestation le 4 mai 1919. Acteur de la Grande guerre du côté des anglais, des français et des américains - des dizaines de milliers de travailleurs ont été enrôlés dans le British Army's Chinese Labour Corps pour creuser les tranchées - les Chinois espéraient récupérer les territoires et droits économiques de l'Allemagne en Chine, mais ceux-ci furent finalement cédés aux Japon. L'invocation de la «Science » et de la « Démocratie » dans les manifestations soulignaient la volonté des acteurs de se servir des méthodes et du savoir occidental afin de recouvrir la souveraineté de la Chine.

4. La présentation du projet ANR en cours est disponible à l'adresse suivante : http:// ifcl.bid (page consultée le 15/10/2015)

5. Sa contribution est suivie d'une courte réponse de François Jullien. 